\title{
Legendre Approximations for Solving Optimal Control Problems Governed by Ordinary Differential Equations
}

\author{
M. El-Kady
}

Department of Mathematics, Faculty of Science, Helwan University, Cairo, Egypt

\begin{abstract}
In this paper Legendre integral method is proposed to solve optimal control problems governed by higher order ordinary differential equations. Legendre approximation method reduced the problem to a constrained optimization problem. Penalty partial quadratic interpolation method is presented to solve the resulting constrained optimization problem. Error estimates for the Legendre appro ximations are derived and a technique that gives an optimal appro ximation of the problems is introduced. Numerical results are included to confirm the efficiency and accuracy of the method.
\end{abstract}

Keywords Spectral Methods, Legendre Polynomials, Optimal Control Problem

\section{Introduction}

Spectral methods using expansion in orthogonal polynomials such as Chebyshev polynomials have proven successful in the numerical approximation of various boundary value problems; see for instance[12]. If these polynomials are used as basis functions, then the rate of decay of the expansion coefficients is determined by the smoothness properties of the function being expanded[17]. This choice of trial functions is responsible for the superior approximation properties of spectral methods when compared with finite difference and finite element methods[2]. For spectral and pseudospectral methods, explicit expressions for the expansion coefficients of the derivatives in terms of the expansion coefficients of the solution are needed. Infinite-horizon Pontryagin's principle has been introduced early in[1]. The authors in[6] introduced a Chebyshev spectral procedure for solving optimal control problems.

$\operatorname{In}[3]$, the author obtained a general formu la when the basis functions are the Ultraspherical polynomials, while Chebyshev and Legendre pseudospectral approximations are used to solve integral and integro-differential equations in[4] and[8], respectively.

It should be mentioned that the study of the existence and the structure of solutions of optimal control problems defined on fin ite intervals has recently been a rapidly growing area of research. See, for example, $[7,9,11,14]$ and the references mentioned therein. A variety of numerical methods for solving infinite horizon variational optimal control problem exists in [15] and[18].

* Corresponding author:

mam el kady@yahoo.com (M. El-Kady)

Publishèd online at http://journal.sapub.org/control

Copyright (C) 2012 Scientific \& Academic Publishing. All Rights Reserved
Some kind of optimal control problems which are governed by ordinary differential equations are discussed in $[5$, 16]. Linear quadratic optimal control problem is solved by using Legendre approximation[10].

The most common approach is to replace the unknowns of the problem by some approximation function and determine the unknowns by minimizing the resulting constrained optimization problem. The time optimal boundary control of a one-dimensional vibrating system subject to a control constraint that prescribes an upper bound for the $L^{2}$-norm of the image of the control function under a Volterra operator[13].

The proposed algorithm describes an alternative technique. The system dynamics can be approximated by transforming the boundary value problem for ord inary differential equations into integral formulas. Start with a Legendre spectral approximation for the highest-order derivative and generate approximations to the lowest-order derivatives through successive integrations. Therefore, the differential and integral expressions that arise in the system dynamics, the performance index and the initial (or boundary) conditions (and even for general multipoint boundary conditions) are converted into algebraic equations with unknown coefficients. This algorithm is of the finite element type and results in static optimization problems with a relatively small number of variables. This means that the optimal control problem is reduced to a parameter static optimization problem, which consis ts of the minimization of an objective function, subject to a system of algebraic constraints that are linear in the state variables, irrespective of whether the dynamic system itself is linear or nonlinear. In such cases, the static optimization problem can be efficiently performed using the penalty partial quadratic interpolation (PPQI) technique[5]. We derived error estimation of this approximation, and introduced an algorith $m$ that gives an optimal approximation of the integrals. 
The paper is organized as follows: In section 2, we introduce mathematical formulation of optimal control problem with linear terminal constraints. In section 3, a Legendre approximate solution is presented. In section 4, error bounds for Legendre method is explained. In section 5, some numerical examples are given to clarify the proposed method and compared with other methods. Finally, in section 6 some remarks and conclusions of the work are presented.

\section{Setting Optimal Control Problems With Linear Terminal Constraints}

Due to the global nature of the s mooth functions, spectral methods are usually global methods, i.e. the value of a derivative at a certain point in space depends on the solution at all the other points in space, and not just the neighboring grid points. Due to this fact, spectral methods usually have a very high order of appro ximation. Spectral convergence meaning that the error is in fact decreasing exponentially as opposed to algebraically as for fin ite-difference methods.

Spectral methods usually give the exact derivative of a function; the only error is due to the truncation to a finite set of smooth functions/coefficients. On the other hand, spectral methods are geometrically less flexible than lower-order methods, and they are usually more complicated to implement. Additionally, the spectral representation of the solution is difficult to combine with sharp gradients, e.g. problems involving shocks and discontinuities.

In the next section, optimal control problems with spectral methods are very adapted and efficient discretization schemes. Now, consider the problem of finding the control $u(t)$ which minimizes the cost functional:

$$
J=h\left(x, x^{(1)}, \ldots, x^{(n-1)}, T\right)+\int_{0}^{T} g\left(x, x^{(1)}, \ldots, x^{(n-1)}, u, \tau\right) d \tau
$$

Subject to

$$
F\left(x, x^{(1)}, \ldots, x^{(n)}, u, \tau\right)=0,0 \leq \tau \leq T
$$

where $x^{(r)}=\frac{d^{r} x}{d x^{r}}, r=1,2, \ldots, n$

and the linear initial constraints,

$$
L\left(x(0), x^{(1)}(0), x^{(2)}(0), \ldots, x^{(n-1)}(0)\right)=0,
$$

and the terminal constraints,

$$
M\left(x(T), x^{(1)}(T), x^{(2)}(T), \ldots, x^{(n-1)}(T)\right)=0,
$$

where the time $T$ is assumed to be fixed, $L$ and $M$ are vector functions of dimension $l$ and $m$, respectively, with $n \leq l+m \leq 2 n$. The state variable $x(\tau) \in R^{N}$, the control variable $u(\tau) \in R^{M}, g(\tau) \in R^{N}$ are real valued continuous functions on $[0, T]$.

To change the time interval $\tau \in[0, T]$ into $t \in[-1,1]$, we have:

$$
t=\frac{2 \tau}{T}-1
$$

Hence the optimal control problem becomes:

Minimize

$J=h\left(x, x^{(1)}, \ldots, x^{(n-1)}, 1\right)+\frac{T}{2} \int_{-1}^{1} g\left(x, x^{(1)}, \ldots, x^{(n-1)}, u, t\right) d t$

Subject to

$$
F\left(x,\left(\frac{2}{T}\right) x^{(1)}, \ldots,\left(\frac{2}{T}\right)^{n} x^{(n)}, u, t\right)=0, \quad-1 \leq t \leq 1,
$$

and the linear initial and terminal constraints,

$$
\begin{gathered}
L\left(x(-1),\left(\frac{2}{T}\right) x^{(1)}(-1), \ldots,\left(\frac{2}{T}\right)^{n} x^{(n)}(-1)\right)=0, \\
M\left(x(1),\left(\frac{2}{T}\right) x^{(1)}(1), \ldots,\left(\frac{2}{T}\right)^{n} x^{(n)}(1)\right)=0,
\end{gathered}
$$

\section{Pseudospectral Legendre Integration Differentiation Matrices}

We present here the Legendre approximations of any function $f(t) \in C^{\infty}[-1,1]$, at $(N+1)$ Legendre-Gauss-Lobatto (LGL) points as follows:

Let $t \in[-1,1]$ such that $\left\{t_{i}:\left(1-t_{i}^{2}\right) P_{N}^{\prime}\left(t_{i}\right)=0, i=0,1, \ldots, N\right\}$.

$$
f_{N}(x)=\sum_{\mathrm{j}=0}^{N} a_{j} P_{j}(t)
$$

where

$$
a_{j} \cong \frac{2 j+1}{N(N-1)} \sum_{k=0}^{N} \frac{P_{j}\left(t_{k}\right) f\left(t_{k}\right)}{\left[P_{N-1}\left(t_{k}\right)\right]^{2}}, j=0,1, \ldots, N
$$

Approximate the integrals of a function as follows:[7]

$$
\left[\int_{-1}^{t} \int_{-1}^{t} \ldots . \int_{-1}^{t} f_{N}(t) d t d t \ldots d t\right]=B^{(n)}\left[\mathbf{P}_{N} f\right],
$$

where the elements of $B^{(n)}=b_{i j}^{(n)}, i, j=0,1, \ldots, N$ are given by: $b_{i k}^{(n)}=\sum_{j=0}^{N} \sum_{m=0}^{[j / 2]} \frac{2 j+1}{N(N-1)\left[P_{N-1}\left(t_{k}\right)\right]^{2}} c_{m}^{j} P_{j}\left(t_{k}\right)\left\{\frac{(j-2 m) !}{(j-2 m+n) !}\left[t_{i}^{j-2 m+n}-(-1)^{j-2 m+n}\right]\right.$

$$
\left.-\sum_{r=1}^{n-1} \sum_{s=0}^{r} \frac{(-1)^{j-2 m+n-r}(j-2 n) ! t_{i}^{s}}{(r-s) ! s !(j-2 m+n-r) !}\right\}, \quad 0 \leq i, k \leq N
$$

and

$$
c=\frac{(-1)(2 n-2 k) !}{2(n-k) !(n-2 k) ! k !} \text {. }
$$

\section{Legendre Approximations for OCP}

Legendre approximations are adopted here to approximate the solution of the problem. We start with Legendre approximations for the highest-order derivative, $x^{(n)}$, and generate approximations to the lowest-order derivatives $x^{(n-1)} ; x^{(n-2)} ; \ldots$ and $x^{(0)}$, through successive integrations of the highest-order derivative.

Suppose that 


$$
x^{(n)}(t)=\Psi(t)
$$

where $\Psi\left(t_{i}\right), i=0,1, \ldots, N$ are some unknowns. By integration, and making use of the given conditions, we get

$$
\begin{gathered}
x^{(n-1)}(t)=\int_{-1}^{t} \Psi(t) d t+c_{0} \\
x^{(n-2)}(t)=\int_{-1}^{t} \int_{-1}^{t} \Psi(t) d t d t+c_{0} t+c_{1} \\
\vdots \\
x(t)=\int_{-1}^{t} \int_{-1}^{t} \ldots \int_{n-1-1}^{t} \int_{\text {times }}^{t} \Psi(t) d t d t \ldots d t+\sum_{r=0}^{n-1} c_{n-r} t^{r}
\end{gathered}
$$

Now we apply our Legendre integral approximation:

$$
\begin{gathered}
x^{(n-1)}\left(t_{i}\right)=\sum_{j=0}^{N} b_{i j} \Psi\left(t_{j}\right)+c_{0}, i=0,1, \ldots, N \\
x^{(n-2)}\left(t_{i}\right)=\sum_{j=0}^{N} b_{i j}^{(2)} \Psi\left(t_{j}\right)+c_{0} t_{i}+c_{1} \\
x\left(t_{i}\right)=\sum_{j=0}^{N} b_{i j}^{(n)} \Psi\left(t_{j}\right)+\sum_{r=0}^{n-1} c_{r} t_{i}^{r}
\end{gathered}
$$

where the constants $c_{r}, r=0,1, . ., n-1$ may be defined fro $m$ the given conditions. Making use of the approximation for the control variable as $u\left(t_{i}\right)=u\left(t_{i}\right)$, the optimal control problem (2.5)-(2.8) are replaced by the following constrained optimization problems:

Minimize

$$
\begin{aligned}
& J=h\left(x, x^{(1)}, \ldots, x^{(n-1)}, 1\right)+\frac{T}{2} \sum_{i=0}^{N} b_{N i} g \\
& \left(\sum_{j=0}^{N} b_{i j}^{(n)} \Psi\left(t_{j}\right)+\sum_{r=0}^{n-1} C_{r} t_{i}^{r}, \ldots, \sum_{j=0}^{N} b_{i j} \Psi\left(t_{j}\right)+C_{0}, u\left(t_{i}\right)\right)
\end{aligned}
$$

Subject to

$$
F\left(\begin{array}{l}
\left\{\sum_{j=0}^{N} b_{i j}^{(n)} \Psi\left(t_{j}\right)+\sum_{r=0}^{n-1} C_{r} t_{i}^{r}\right\}, \ldots,\left(\frac{2}{T}\right)^{n-1}, \\
\left\{\sum_{j=0}^{N} b_{i j} \Psi\left(t_{j}\right)+C_{0}\right\},\left(\frac{2}{T}\right)^{n} \Psi\left(t_{i}\right), u\left(t_{i}\right)
\end{array}\right)=0
$$

The constrained optimization problem is then takes the form:

$$
\begin{aligned}
& \text { Minimize } J=J[\boldsymbol{\alpha}], \\
& \text { Subject to } F[\boldsymbol{\alpha}]=0,
\end{aligned}
$$

where $\boldsymbol{\alpha}=\left[\Psi\left(t_{0}\right), \Psi\left(t_{1}\right), \ldots, \Psi\left(t_{N}\right), u\left(t_{0}\right), u\left(t_{1}\right), \ldots, u\left(t_{M}\right)\right]$.

The problem (4.6)-(4.7) is solved by using penalty partial quadratic interpolation technique[5]. We therefore use:

$$
\left(\sum_{i=0}^{N} F_{i}^{2}\right)^{1 / 2}<\varepsilon
$$

to decide whether the computed solution in close enough to the optimal solution.

\section{Error Estimation}

In this section, an estimation of the error bound of the Legendre integration method is presented in the flowing theorem.

\section{The orem (5.1)[8]}

Let $f(t)$ be approximated by Legendre polynomials, then there exists a number $\xi=\xi(t) \in[-1,1]$ such that

$$
\begin{gathered}
f(t)=\sum_{k=0}^{N} a_{k} P_{k}(t), \\
\int_{-1-1}^{t_{i}} \int_{\text {n-times }}^{t} \ldots \int_{-1}^{t} f(t) d t d t \ldots d t=\sum_{k=0}^{N} b_{i k}^{(n)} f\left(t_{k}\right)+E_{n}\left(t_{i}, \xi\right),
\end{gathered}
$$

where

$$
E_{n}\left(t_{\mathrm{i}}, \xi\right)=\frac{f^{(\mathrm{N}+1)}(\xi)}{(\mathrm{N}+1) ! K_{N+1}} \int_{-1}^{t_{i}} \int_{-1}^{t} \ldots \int_{-1}^{t} P_{N+1}(t) \mathrm{d} t \mathrm{~d} t \ldots \mathrm{d} t
$$

and

$$
K_{N+1}=\frac{(2 N+1) !}{2^{N}(N+1) ! N !} .
$$

The following theorem gives the error bounds of the system dyna mic.

\section{Theorem (5.2)}

Assume the OCP (2.5)-(2.8) is approximated by Legendre approximations and assuming that $x^{(n+N+1)}(t)$ is bounded i.e.

$$
\left\|x^{(n+N+1)}(t)\right\| \leq D,
$$

then there exists a number $\xi(t) \in[-1,1]$ such that

$$
E\left(t_{i}, \xi\right) \leq \frac{D}{(N+1) ! K_{N+1}} \int_{-1}^{t_{i}} \int_{-1}^{t} \ldots . \int_{-1}^{t} \mathrm{P}_{N+1}(t) d t d t \ldots d t,
$$

$$
\begin{gathered}
E_{F}\left(t_{i}\right)=F\left(\sum_{j=0}^{N} b_{i j}^{(n)} \Psi\left(t_{j}\right)+E_{n}\left(t_{i}, \xi\right)+\sum_{r=0}^{n-1} C_{r} t_{i}^{r},\right. \\
\sum_{j=0}^{N} b_{i j}^{(n-1)} \Psi\left(t_{j}\right)+E_{n-1}\left(t_{i}, \xi\right)+\sum_{r=0}^{n-2} C_{r} t_{i}^{r}, \\
\left.\ldots, \sum_{j=0}^{N} b_{i j} \Psi\left(t_{j}\right)+C_{0}+E_{1}\left(t_{i}, \xi\right), u\left(t_{i}\right)\right) \\
-F\left(\sum_{j=0}^{N} b_{i j}^{(n)} \Psi\left(t_{j}\right)+\sum_{r=0}^{n-1} C_{r} t_{i}^{r}, \sum_{j=0}^{N} b_{i j}^{(n-1)} \Psi\left(t_{j}\right)+\sum_{r=0}^{n-2} C_{r} t_{i}^{r}\right. \\
\left., \ldots, \sum_{j=0}^{N} b_{i j} \Psi\left(t_{j}\right)+C_{0}, u\left(t_{i}\right)\right) .
\end{gathered}
$$

Proof:

Let $E\left(t_{i}, \xi\right)$ denote the error in approximation $x\left(t_{i}\right)$ with (4.3), namely

$$
E\left(t_{i}\right)=\int_{-1}^{t_{i}} \int_{-1}^{t} \ldots . \int_{-1}^{t} \Psi(t) d t d t \ldots d t-\sum_{j=0}^{N} b_{i j}^{(n)} \Psi\left(t_{j}\right),
$$


then, making use of (5.1) and (5.2), the error in the approximation (4.3) can be written as:

$$
\begin{aligned}
E\left(t_{i}, \xi\right) & =\frac{\Psi^{(N+1)}(\xi)}{(N+1) ! K_{N+1}} \int_{-1}^{t_{i}} \int_{-1}^{t} \ldots . . \int_{-1}^{t} \mathrm{P}_{N+1}(t) d t d t \ldots d t \\
& =\frac{x^{(\mathrm{n}+N+1)}(\xi)}{(N+1) ! K_{N+1}} \int_{-1}^{t_{i}} \int_{-1}^{t} \ldots . \int_{-1}^{t} \mathrm{P}_{N+1}(t) d t d t \ldots d t
\end{aligned}
$$

Thus, making use of (5.3), we get

$$
E\left(t_{i}, \xi\right) \leq \frac{D}{(N+1) ! K_{N+1}} \int_{0}^{t_{i}} \int_{0}^{t} \ldots . . \int_{0}^{t} \mathrm{P}_{N+1}(t) d t d t \ldots d t .
$$

The original constraint (2.6) in view of (4.2) beco mes:

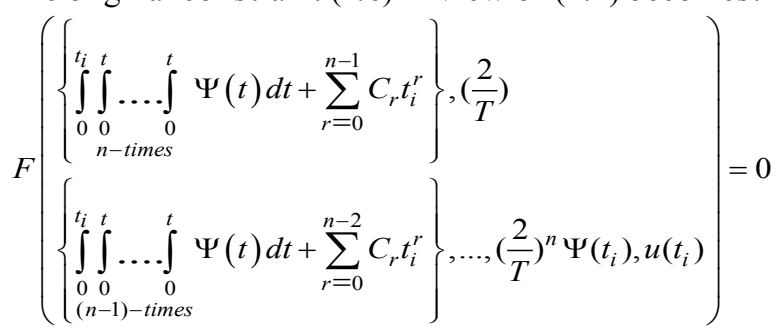

Making use of (5.1) then,

$$
F\left(\sum_{j=0}^{N} b_{i j}^{(n)} \Psi\left(t_{j}\right)+E_{n}\left(t_{i}, \xi\right)+\sum_{r=0}^{n-1} C_{r} t_{i}^{r},\right.
$$

$\left.\ldots,\left(\frac{2}{T}\right)^{n-1} \sum_{j=0}^{N} b_{i j} \Psi\left(t_{j}\right)+E_{1}\left(t_{i}, \xi\right)+C_{0},\left(\frac{2}{T}\right)^{n} \Psi\left(t_{j}\right), u\left(t_{i}\right)\right)=0$

Subtracting (4.5) from(5.7), we obtain

$$
\begin{gathered}
E_{F}\left(t_{i}\right)=F\left(\sum_{j=0}^{N} b_{i j}^{(n)} \Psi\left(t_{j}\right)+E_{n}\left(t_{i}, \xi\right)+\sum_{r=0}^{n-1} C_{r} t_{i}^{r},\right. \\
\left.\ldots,\left(\frac{2}{T}\right)^{n-1} \sum_{j=0}^{N} b_{i j} \Psi\left(t_{j}\right)+E_{1}\left(t_{i}, \xi\right)+C_{0},\left(\frac{2}{T}\right)^{n} \Psi\left(t_{j}\right), u\left(t_{i}\right)\right)- \\
F\left(\begin{array}{l}
\left\{\sum_{j=0}^{N} b_{i j}^{(n)} \Psi\left(t_{j}\right)+\sum_{r=0}^{n-1} C_{r} t_{i}^{r}\right\}, \ldots,\left(\frac{2}{T}\right)^{n-1}, \\
\left\{\sum_{j=0}^{N} b_{i j} \Psi\left(t_{j}\right)+C_{0}\right\},\left(\frac{2}{T}\right)^{n} \Psi\left(t_{i}\right), u\left(t_{i}\right)
\end{array}\right) .
\end{gathered}
$$

with $E_{n}\left(t_{i}, \xi\right)$ is defined in (5.2).

\section{Numerical Examples and Application}

Now, we consider the following problems to show the effectiveness of our technique.

\section{Example 1:}

Among all piecewise differentiable control variab les, find the optimal control $u(t)$ which minimizes

$$
J=\int_{0}^{1}\left[x^{2}(\tau)+x^{\prime 2}(\tau)+0.005 u^{2}(\tau)\right] d \tau
$$

Subject to:

$$
\begin{gathered}
x^{\prime \prime}(\tau)+x^{\prime}(\tau)-u(\tau)=0, \\
x(\tau)-8(\tau-0.5)^{2}+0.5 \leq 0 . \\
x(0)=0, \quad x^{\prime}(0)=-1
\end{gathered}
$$

The first step in solving this problem by the proposed method is to transform the time interval into $t \in[-1,1]$. At the end, this will lead to the following problem.

Minimize

$$
J=\frac{1}{2} \sum_{i=0}^{N} b_{N i}\left(\begin{array}{l}
{\left[\sum_{k=0}^{N} b_{i k}^{(2)} \Psi_{k}-t_{i}\right]^{2}} \\
+4\left[\sum_{k=0}^{N} b_{i k} \Psi_{k}-1\right]^{2}+0.005 u^{2}\left(t_{i}\right)
\end{array}\right),
$$

Subject to:

$$
4 \Psi\left(t_{i}\right)-\left[\sum_{k=0}^{N} b_{i k} \Psi_{k}-1\right]-u\left(t_{i}\right)=0 .
$$

We approximate the inequality constraint by adding slack variables:

$$
\left(\sum_{k=0}^{N} b_{i k}^{(2)} \Psi_{k}-t_{i}\right)-2 t_{i}^{2}+0.5+A_{N+i}^{2}=0 .
$$

Solving this problem (6.9)-(6.11) by using the proposed method by $9^{\text {th }}$ order Legendre, we find the optimal value is $J^{*}=0.71426412$. The optimal state and control are shown in Figs. (1) and (2), respectively. The author in[8] used of cell averaging Chebyshev method by $9^{\text {th }}$ order Chebyshev for solve this examp le and get $J^{*}=0.74096103$.

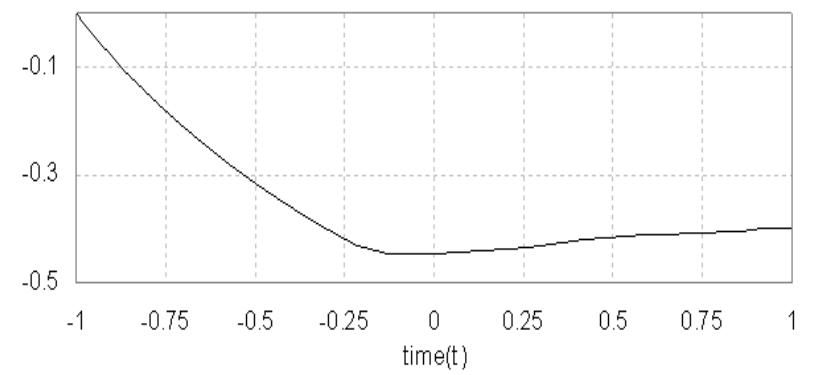

Figure 1. state variable $x(t)$ of example(1)

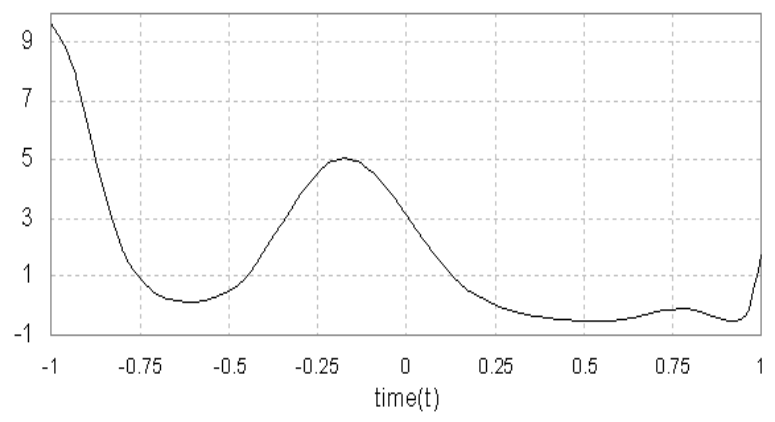

Figure 2. control varaible $u(t)$ of example(1)

\section{Example 2: The Controlled Linear Oscillator}

Consider the optimal control problem of a liner oscillator the performance index 


$$
J=\frac{1}{2} \int_{-T}^{0} u^{2}(\tau) d \tau
$$

is minimized over all admissible control functions $u(\tau)$.

Subject to the differential equation

$$
\ddot{x}(\tau)+\omega^{2} x(\tau)=u(\tau), \quad-T \leq \tau \leq 0
$$

with the boundary conditions

$$
x(-T)=x_{0}, \quad x(0)=0
$$

The problem can be converted to the following constrained optimization problem: Minimize

$$
J=\frac{T}{4} \sum_{i=0}^{N} b_{N i} u^{2}\left(t_{i}\right)
$$

Subject to

$$
\begin{aligned}
& \Psi\left(t_{i}\right)-\frac{T^{2}}{4} \\
& \left(-\omega^{2}\left\{\sum_{k=0}^{N} b_{i k}^{(2)} \Psi\left(t_{k}\right)+\frac{1}{2} x_{0}\left(1-t_{i}\right)-\frac{1}{2} x_{0}\left(1+t_{i}\right) \sum_{k=0}^{N} b_{N k}^{(2)} \Psi\left(t_{k}\right)\right\}+u\left(t_{i}\right)\right)=0
\end{aligned}
$$

At $\omega=1, T=2$ and $x_{0}=0.5$, we get the optimal result $J^{*}=0.18485854$ with $N=18$. Table (1) gives the optimal values of the cost functional $J^{*}$ for different values of $N$. The state and controlvariables are shown in Figs. (3) and (4), respectively.

Table 1. $J^{*}$ of present method with other methods

\begin{tabular}{ccc}
\hline Methods & $N, M$ & $J^{*}$ \\
\hline \multirow{2}{*}{ Van Dooren[19] } & $\mathrm{M}=4$ & 0.18491700 \\
& $\mathrm{M}=7$ & 0.18485854 \\
& $\mathrm{M}=10$ & 0.18485854 \\
Elnagar[11] & $\mathrm{M}=5$ & 0.18485790 \\
& $\mathrm{M}=6$ & 0.18485854 \\
El-Gindy[5] & $\mathrm{M}=7, \mathrm{~N}=11$ & 0.18485122 \\
& $\mathrm{M}=11, \mathrm{~N}=15$ & 0.18485124 \\
Present method & $\mathrm{N}=8$ & 0.18485851 \\
& $\mathrm{~N}=10$ & 0.18485851 \\
& $\mathrm{~N}=12$ & 0.18485851 \\
\hline
\end{tabular}

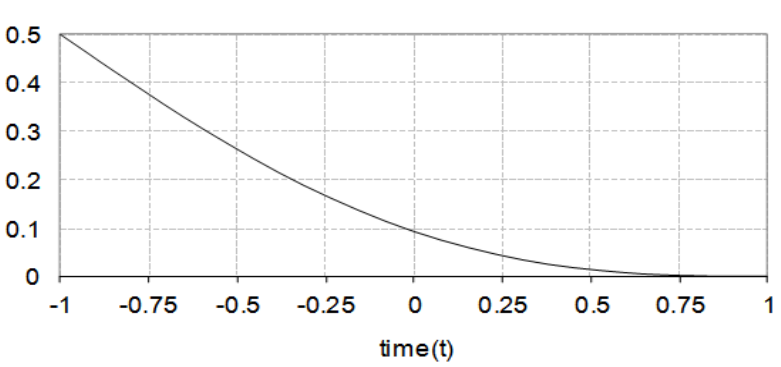

Figure 3. State varible $x(t)$ of example (2)

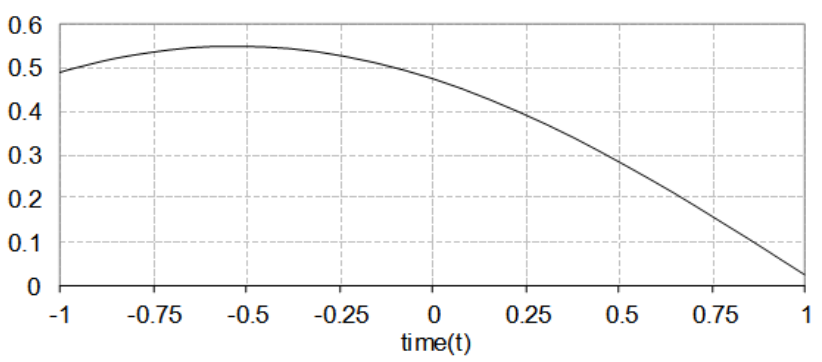

Figure 4. control variable $u(t)$ of example (2)
Example (3) Find a suitable control for minimization of the following optimal control problem[24]:

Minimize

$$
J=0.5 \int_{-1}^{1}\left[x_{1}^{2}(t)+x_{2}^{2}(t)+u^{2}(t)\right] d t
$$

Subject to:

$$
\begin{aligned}
& 2 \dot{x}_{1}(t)=x_{2}(t)+u(t) \\
& 2 \dot{x}_{2}(t)=u(t),
\end{aligned}
$$

with the boundary conditions:

$$
x_{1}(-1)+x_{2}(-1)=3, x_{2}(1)=1
$$

By apply the proposed method; the optimal values of state and control given in Table (2). Table (3) shows that the presented Legendre approximations are more efficiency than the method in[16].

Table 2. Observed state $\mathrm{x}(\mathrm{t})$ and control $\mathrm{u}(\mathrm{t})$ variables for Example(3)

\begin{tabular}{|c|c|c|c|}
\hline $\mathrm{T}$ & $\chi_{1}(\mathrm{t})$ & $\chi_{2}(\mathrm{t})$ & $\mathrm{u}(\mathrm{t})$ \\
\hline-1.000 & 1.21 & 1.79 & -2.422 \\
\hline-0.951 & 1.195 & 1.732 & -2.32 \\
\hline-0.809 & 1.158 & 1.578 & -2.038 \\
\hline-0.588 & 1.118 & 1.375 & -1.635 \\
\hline-0.309 & 1.099 & 1.179 & -1.179 \\
\hline 0.000 & 1.123 & 1.033 & -0.725 \\
\hline 0.309 & 1.196 & 0.953 & -0.307 \\
\hline 0.588 & 1.309 & 0.936 & 0.051 \\
\hline 0.809 & 1.435 & 0.957 & 0.331 \\
\hline 0.951 & 1.534 & 0.987 & 0.512 \\
\hline 1.000 & 1.571 & 1 & 0.575 \\
\hline
\end{tabular}

Table 3. Comparison with results in Ref.[16]

\begin{tabular}{|c|c|c|}
\hline $\mathrm{N}$ & $\begin{array}{c}J^{*} \\
\text { Present Method }\end{array}$ & $\begin{array}{c}J^{*} \\
\text { Ref.[16] }\end{array}$ \\
\hline 8 & 4.20767791 & 4.20767795 \\
\hline 10 & 4.20767791 & 4.20767793 \\
\hline
\end{tabular}

Table 4. State and control variables for Example (4)

\begin{tabular}{|c|c|c|c|}
\hline $\mathrm{t}$ & $\chi_{1}(\mathrm{t})$ & $\chi_{2}(\mathrm{t})$ & $\mathrm{u}(\mathrm{t})$ \\
\hline-1.000 & -5 & -5 & 2.767 \\
\hline-0.951 & -5.255 & -3.312 & 5.568 \\
\hline-0.809 & -5.432 & 1.043 & 3.152 \\
\hline-0.588 & -4.627 & 4.081 & 0.881 \\
\hline-0.309 & -3.101 & 4.388 & 0.405 \\
\hline 0.000 & -1.522 & 3.68 & -0.591 \\
\hline 0.309 & -0.321 & 2.528 & -1.097 \\
\hline 0.588 & 0.423 & 1.845 & -0.585 \\
\hline 0.809 & 0.91 & 1.736 & -0.132 \\
\hline 0.951 & 1.226 & 1.846 & 0.089 \\
\hline 1.000 & 1.341 & 1.892 & 0.057 \\
\hline
\end{tabular}

Example (4) Find a suitable control for minimization of the following optimal control problem:

Minimize

$$
J=1.25 \int_{-1}^{1}\left[x_{1}^{2}(t)+u^{2}(t)\right] d t
$$

Subject to:

$$
0.8 \dot{x}_{1}(t)=x_{2}(t)
$$




$$
0.8 \dot{x}_{2}(t)=-x_{1}(t)+4 u(t)+1.4 x_{2}(t)-0.14 x_{2}^{3}(t)
$$

and

$$
x_{1}(-1)=-5, x_{2}(-1)=-5
$$

The optimal cost is $J^{*} \approx 29.37170568$ as given in[9]. Table (4) shows the state and control variables as computed by the proposed method.

Table 5. Comparison with results in Ref.[11]

\begin{tabular}{|c|c|}
\hline Methods & $J^{*}$ \\
\hline Ref.[11] & 29.4081 \\
\hline Present method with N=10 & 29.2928 \\
\hline
\end{tabular}

The major advantages of this method is that, we can deal directly with the highest-order derivatives in the differential equation without transforming it to a system of first order, and that will reduce the number of the unknowns. The tables show that the suggested technique is quite reliable. The methods produce an accurate solution at small number of nodes. The comparis on of the maximum absolute error resulting from the proposed method and those obtained by [5],[11] and[19] show favourable agreement and always it is more accurate than these treatments.

\section{Conclusions}

The basic idea of our present method is to transform the optimal control problems governed by ordinary differential equations to a constrained optimization problem, by using Legendre approximation method. We solve the resulting constrained optimization problem since it is easier than solving the original problem. Here we used (PPQI) method, which may be more suitable in such case, where the number of constraints is increases. Finally, the method has been extended to the linear and nonlinear optimal control problems.

\section{REFERENCES}

[1] J. Blot, Infinite-horizon Pontryagin principles without invertibility, J. Nonlinear Convex Anal. 10 (2009) 177-189.

[2] C. Canuto, M. Y. Hussaini, A. Quarteroni and Zang, T. A. "Spectral Methods in Fluid Dynamic" Springer-Verlag, New York Inc., 1988.

[3] E.H. Doha, "The Ultraspherical Coefficients of Moments of a General Order Derivatives of an Infinitely Differentiable Function "J. Comput. and Appl. Math.,89, (1998)53-72.

[4] S.E. EL-gendi, "Cheby shev Solutions of Differential, Integral, and Integro -Differential Equations " Computer J., 12, (1969) 282-287.
[5] T.M. E1-Gindy \& M.S. Salim, "Penalty function with partial quadratic interpolation technique in the constrained optimization problems", Journal of Institute of Math. Computer Sci. 3 (1), (1990) 85-90.

[6] T. M. El-Gindy, T. M., El-Hawary, H. M., Salim, M. S. and El-Kady, M. "A Chebyshev Approximation for Solving Optimal Control Problems", Computers Math. Applic. 29 (6), (1995) 35-45.

[7] V. Lykina, S. Pickenhain, M. Wagner, Different interpretations of the improper integral objective in an infinite horizon control problem, J. Math. Anal. Appl. 340 (2008) 498-510.

[8] M. El-Kady and M. Biomy "Efficient Legendre Pseudospectral Method for Solving Integral and Integro-Differential Equations" Commun Nonlinear Sci Numer Simulat 15 (2010) 1724-1739.

[9] M. El-Kady and M. Biomy, "Interactive Cheby shev-Legendre Algorithm for Lin ear Quadratic Optimal Regulator Systems", Int. J. of Wavelets, Multiresolution and Information Proc., Vol. 9, No. 3, (2011) 1-25.

[10] M. El-Kady, Efficient reconstructed Legendre algorithm for solving linear-quadratic optimal control problems Applied Mathematics Letters 25 (2012) 1034-1040

[11] G.M. Elnagar, "State-control Spectral Chebyshev Parameterization for Linearly Constrained Quadratic Optimal Control Problems", J. Comput. And Applied Mathes. 97, (1997), pp 19-40.

[12] D. Gottlieb, and Orszag, S.A. "Numerical Analysis of Spectral Methods : Theory and Application" CBMS-NSF Regional Conference Series in Applied Mathematics , 26, Philadelphia: SIAM, 1977

[13] G. Martin "A Newton Method for the Computation of Time-Optimal Boundary Controls of One-Dimensional Vibrating Systems" Journal of Comput. and Applied Math. 114 (2000) 103-119.

[14] S. Mashayekhi, Y. Ordokhani, M. Razzaghi, "Hybrid functions approach for nonlinear constrained optimal control problems",Commun Nonlinear Sci Numer Simulat 17 , (4) (2012) 1831-1843

[15] A.B. Malinowska, N. Martins, D.F.M. Torres, Transversality conditions for infinite horizon variational problems on time scales, Optim. Lett. 5 (2011) 41-53.

[16] A. A. Salama, "Numerical methods based on extended one-step methods for solving optimal control problems", Appl. Math. Comput. 183 (2006) 243-250.

[17] Szegö, "Orthogonal Polynomials", Am. Math. Soc. Colloq. Pub. ,23, 1985

[18] E. Ocana Anaya, P. Cartigny, P. Loisel, Singular infinite horizon calculus of variations. Applications to fisheries management, J. Nonlinear Convex Anal. 10 (2009) 157-176.

[19] R. Van Dooren, J. and Vlassenbroeck, "A Chebyshev Technique for Solving Nonlinear Optimal Control Problems", IEEE Trans. Automat. Contr., 33 (4), (1988) 333-339. 